\title{
EPR Study of Chromium Ions Doped Gallium Borate
}

\author{
A.A. Prokhorov ${ }^{a, *}$, L.F. Chernush ${ }^{b}$, R. Minikayev ${ }^{c}$, J. LAnČOK ${ }^{a}$ And A.D. Prokhorov ${ }^{b}$ \\ ${ }^{a}$ Institute of Physics AS CR, Na Slovance 2, 18221 Prague, Czech Republic \\ ${ }^{b}$ A.A. Galkin Donetsk Institute for Physics and Engineering, R. Luxembourg 72, 83114 Donetsk, Ukraine \\ ${ }^{c}$ Institute of Physics, Polish Academy of Sciences, Aleja Lotnikow 32/46, PL-02668, Warsaw, Poland
}

(Received July 22, 2019; in final form September 9, 2019)

\begin{abstract}
New data on the state of $\mathrm{Cr}^{3+}$ ion impurities in a single crystal of $\mathrm{YGa}_{3}\left(\mathrm{BO}_{3}\right)_{4}$ were obtained from analysis of the electron paramagnetic resonance spectra. Crystal structure, lattice parameters, and atomic position of this crystal were studied by X-ray powder diffraction method. The $\mathrm{Cr}^{3+}$ ions are found to substitute for the trivalent gallium ions. The spectra of $\mathrm{Cr}^{3+}$ ions located at three magnetically nonequivalent sites that rotated through $120^{\circ}$ are registered. The obtained parameters of spin Hamiltonian of the $\mathrm{Cr}^{3+}$ ions in the $\mathrm{YGa}_{3}\left(\mathrm{BO}_{3}\right)_{4}$ single crystal were analyzed. Crystal structure, lattice parameters, and atomic position of this crystal are studied by X-ray powder diffraction method.
\end{abstract}

DOI: 10.12693/APhysPolA.136.947

PACS/topics: EPR, RAB, X-ray diffraction, multiferroics, rare-earth, aluminum borates, gallium borates, spin Hamiltonian parameters, superposition model, low-spin state

\section{Introduction}

Rare-earth borates $\mathrm{RM}_{3}\left(\mathrm{BO}_{3}\right)_{4}$ (where $\mathrm{R}=\mathrm{Y}, \mathrm{La}-\mathrm{Lu}$; $\mathrm{M}=\mathrm{Al}, \mathrm{Ga}, \mathrm{Fe}, \mathrm{Cr}$ ) belong to trigonal crystallographic system and for a new class of multiferroics, in which magnetic, electrical, and order parameters coexist. Rareearth aluminum borates and gallium borates combine good luminescent and pronounced nonlinear optical properties. They belong to the new generation of materials for lasers.

High concentration of doping ions in aluminum borates is not accompanied by concentration quenching. This fact as well as distinctive physical and chemical properties contribute to promising application as media for solid-state lasers. By using single-crystal rareearth borates, effective transformation of the frequency of laser radiation can be performed that substantially exceeds analogous parameters of the known media. Crystals of borates doped by ytterbium allow generation of femtosecond laser impulses. The erbium-doped crystals provide laser irradiation characterized by the wavelength of $1.5 \mu \mathrm{m}$ that finds an application in medicine [1-6].

Interaction between the iron ions in quasi-onedimensional chains in $\mathrm{ReFe}_{3}\left(\mathrm{BO}_{3}\right)_{4}$ results in antiferromagnetic ordering in the range of $30-37 \mathrm{~K}$ [7]. Weak spin-spin interaction between the $\mathrm{Tb}^{3+}$ ions forms magnetic ordering in the $\mathrm{TbAl}_{3}\left(\mathrm{BO}_{3}\right)_{4}$ crystal at the temperature of $0.68 \mathrm{~K}[8]$. An important result is magnetoelectric effect found in the $\mathrm{HoAl}_{3}\left(\mathrm{BO}_{3}\right)_{4}$ crystals that is characterized by record-breaking electrical polarization in magnetic field in multiferroics [9].

The study of the properties of the described materials was mostly concentrated on the crystals containing

*corresponding author; e-mail: andaprokhorov@gmail.com ions of $\mathrm{Al}\left(\mathrm{ReAl}_{3}\left(\mathrm{BO}_{3}\right)_{4}\right)$, $\mathrm{Fe}\left(\mathrm{ReFe}_{3}\left(\mathrm{BO}_{3}\right)_{4}\right)$ and $\mathrm{Cr}\left(\mathrm{ReCr}_{3}\left(\mathrm{BO}_{3}\right)_{4}\right)$. A series of works devoted to studies of EPR spectra in aluminum borates doped with $\mathrm{Mn}^{2+}$ [10-13], $\mathrm{Eu}^{2+}[14], \mathrm{Ru}^{3+}[15], \mathrm{Cr}^{3+}[13,16-20]$ and $\mathrm{Ti}^{3+}[21]$, as well as those doped with $\mathrm{Ce}^{3+}$ and $\mathrm{Yb}^{3+}[22], \mathrm{Er}^{3+}$ and $\mathrm{Nd}^{3+}[23], \mathrm{Co}^{2+}[24], \mathrm{Gd}^{3+}[25-27]$ and $\mathrm{Dy}^{3+}$ [28] rare-earth ions was published. Much less attention was paid to the crystals with $\mathrm{M}=\mathrm{Ga}$ $\left(\operatorname{ReGa}_{3}\left(\mathrm{BO}_{3}\right)_{4}\right)$ that can demonstrate no less interesting properties. A number of works are known that deal with structure tests, crystal growth, and spectroscopy of gallium borates [29-32]. In the crystals of $\mathrm{HoGa}_{3}\left(\mathrm{BO}_{3}\right)_{4}$, high magnetoelectric effect was found [33]. Thus, the study of spectroscopic, non-linear, and low-temperature properties of the crystals considered is of both scientific and practical interest.

\section{Experimental procedure}

For sample structural properties confirmation, the X-ray diffraction patterns were collected with help of a laboratory diffractometer X'Pert Pro Alpha1 MPD (Panalytical), equipped with $\mathrm{Cu} K_{\alpha}$ X-ray tube, a $\mathrm{Ge}(111)$ primary beam monochromator and X'Celerator - a linear position-sensitive semiconductor detector. Crystal structures of the sample were refined by the Rietveld method. For this procedure Fullprof.2k program [34] was used.

The EPR spectra were measured using X-band radiation, within the temperature range of 5-300 K. Continuous wave EPR spectra were measured by using a Bruker X-/Q-band E580 FT/CW ELEXSYS spectrometer. For the measurements, the ER 4122 SHQE Super X High-Q cavity with TE011mode was used. The experimental parameters were: microwave frequency $9.875 \mathrm{GHz}$, microwave power $1.500 \mathrm{~mW}$, modulation frequency $100 \mathrm{kHz}$, modulation amplitude $0.2 \mathrm{mT}$, and the conversion time $60 \mathrm{~ms}$. 


\section{Results and discussion}

Analysis of the X-ray diffraction pattern indicates that the $0.1 \% \mathrm{Cr}$-doped $\mathrm{YGa}_{3}\left(\mathrm{BO}_{3}\right)_{4}$ sample crystallizes in the huntite $\left(\mathrm{Mg}_{3} \mathrm{Ca}\left(\mathrm{CO}_{3}\right)_{4}\right)$ structure with the space group R32. In the sample, small amount ( $\sim 7 \mathrm{wt} \%)$ of $\beta-\mathrm{Ga}_{2} \mathrm{O}_{3}$ needle-shaped crystallites were detected. Structure of Cr-doped $\mathrm{YGa}_{3}\left(\mathrm{BO}_{3}\right)_{4}$ is plotted in Fig. 1 . The crystallographic model implemented in the Rietveld refinement procedure is described in [35] and [36] for similar (aluminum based) borate. The atomic positions and isotropic atomic displacement parameters $\left(B_{\text {iso }}\right)$ fitting results are located in Table I. Graphical result of fitting is plotted in Fig. 2. Lattice parameters of the $\mathrm{YGa}_{3}\left(\mathrm{BO}_{3}\right)_{4}: 0.1 \% \mathrm{Cr}$ phase and their ratio (here $a=9.4491(1) \AA, \quad c=7.4546(1) \AA, \quad c / a=0.78892(3))$ are close to previously reported values for undoped material, i.e.., [31] $(a=9.459(4) \AA, c=7.438(3) \AA$, $c / a=0.786(1)), \quad$ and $[34](a=9.428(3) \AA$, $c=7.433(2) \AA, \quad c / a=0.7884(7)) . \quad$ The slight disparities in the lattice parameters absolute values can be related to different crystals growth method, but the $c / a$ ratios are adequately compatible for all data.

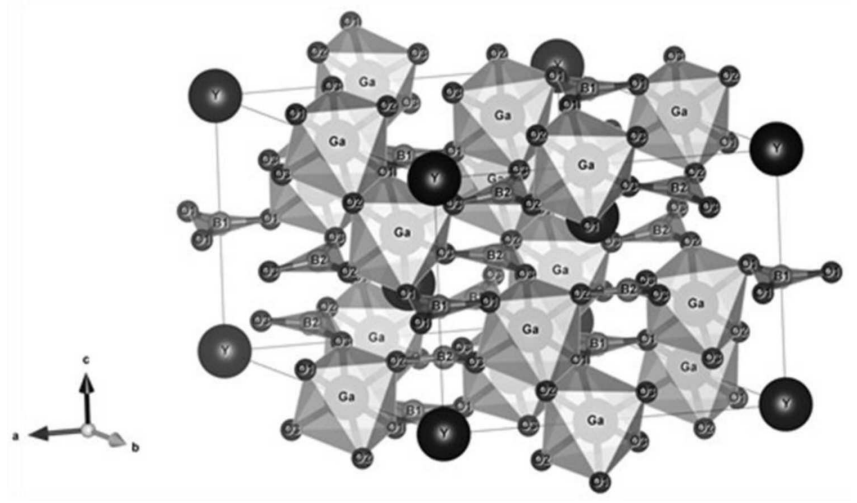

Fig. 1. Structure of Cr-doped $\mathrm{YGa}_{3}\left(\mathrm{BO}_{3}\right)_{4}$.

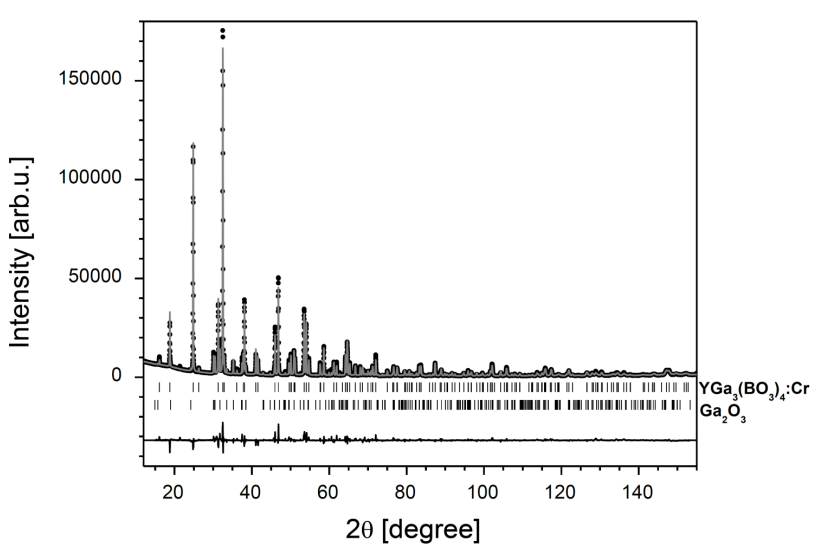

Fig. 2. The Rietveld refinement result for $\mathrm{YGa}_{3}\left(\mathrm{BO}_{3}\right)_{4}$ : $0.1 \%$ Cr sample.
TABLE I

Atomic positions and isotropic atomic displacements in the structure of Cr-doped $\mathrm{YGa}_{3}\left(\mathrm{BO}_{3}\right)_{4}$.

\begin{tabular}{c|c|c|c|c}
\hline \hline \multirow{2}{*}{ Atom } & \multicolumn{3}{|c|}{ Atomic positions } & \multirow{2}{*}{$B_{\text {iso }}$} \\
\cline { 2 - 4 } & $x$ & $y$ & $z$ & $0.740(30)$ \\
$\mathrm{Y}$ & 0 & 0 & 0 & $0.673(26)$ \\
$\mathrm{Ga} / \mathrm{Cr}$ & $0.5505(1)$ & 0 & 0 & $0.28(37)$ \\
$\mathrm{B}(1)$ & 0 & 0 & 0.5 & $1.29(19)$ \\
$\mathrm{B}(2)$ & $0.449(1)$ & 0 & 0.5 & $0.86(14)$ \\
$\mathrm{O}(1)$ & $0.8638(6)$ & 0 & 0.5 & $0.40(11)$ \\
$\mathrm{O}(2)$ & $0.5861(6)$ & 0 & 0.5 & $1.47(11)$ \\
$\mathrm{O}(3)$ & $0.4497(6)$ & $0.1420(5)$ & $0.5088(7)$ &
\end{tabular}

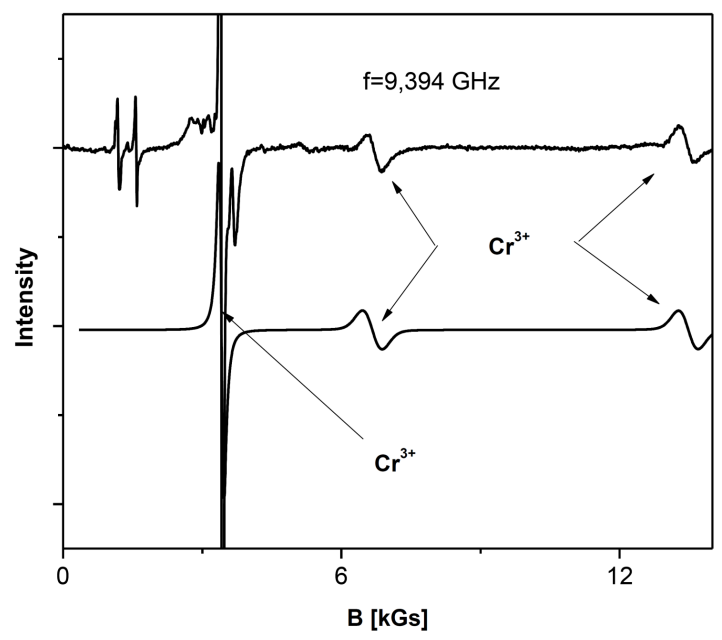

Fig. 3. The EPR spectrum of the $\mathrm{Cr}^{3+}$ ion in a $\mathrm{YGa}_{3}\left(\mathrm{BO}_{3}\right)_{4}$ crystal at a frequency of $f=9.384 \mathrm{GHz}$, $B \quad \| \quad C_{3} . \quad 1$ - experimental spectrum recording; 2 - spectrum calculated by the parameters.

Magnetic properties of the $\mathrm{YGa}_{3}\left(\mathrm{BO}_{3}\right)_{4}: x \% \mathrm{Cr}$ samples $(x=0.1,0.2)$ were studied using electron paramagnetic resonance (EPR).

An ion of trivalent chromium $\mathrm{Cr}^{3+}$ has $3 d^{3}$ electron configuration. The fundamental term ${ }^{4} \mathrm{~F}(L=3$, $S=3 / 2$ ) is split in the crystal field, so the lowest energy is associated with orbital singlet $\Gamma_{2}$ composed by two Kramer's doublets that are also split in the crystal fields characterized by the symmetry lower than the cubic one. The transitions between the levels of the described quartet with the selection rule $\Delta m_{s}= \pm 1$ are registered in the EPR spectrum.

In Fig. 3, the EPR spectra observed at the frequencies $f=9.384 \mathrm{GHz}$ in $B \| C_{3}$ at the temperature of $293 \mathrm{~K}$ are demonstrated. Three EPR spectra are registered that are magnetically non-equivalent but coinciding when the external magnetic field is directed along the $C_{3}$ axis. This fact allows to conclusion that $\mathrm{Cr}^{3+}$ ions substitutes for $\mathrm{Ga}^{3+}$ ions $\mathrm{YGa}_{3}\left(\mathrm{BO}_{3}\right)_{4}$. An analogous substitution takes place in aluminum borates $\mathrm{YAl}_{3}\left(\mathrm{BO}_{3}\right)_{4}, \mathrm{EuAl}_{3}\left(\mathrm{BO}_{3}\right)_{4}$, and 
$\mathrm{TmAl}_{3}\left(\mathrm{BO}_{3}\right)_{4} \quad[16-18,20]$. The crystal lattice site, in which the $\mathrm{Cr}^{3+}$ ion is located belongs to monoclinic symmetry and has the only symmetry element, the $C_{2}$ axis, which is perpendicular to the crystal face. One of the axes of the spectrum $(Y)$ is directed along this axis. The other two spectral axes may have an arbitrary direction and do not coincide with the symmetry elements of the crystal. A similar pattern was observed in the spectrum of the $\mathrm{Ti}^{3+}[21]$ ion and in the spectrum of the $\mathrm{Co}^{2+}[24]$ ion in yttrium aluminum borates. Monoclinic symmetry can lead to the appearance of low symmetry effects in the EPR spectra, which are manifested in the mismatch of the transition axes corresponding to different pairs of energy levels of the paramagnetic center, in the mismatch of the axes of the $g$-tensor and the hyperfine splitting tensor, in the asymmetry of the angular dependence of the magnetic spectrum.

When studying the EPR spectrum of the $\mathrm{Cr}^{3+}$ ion, we did not observe the low-symmetry effects listed above (possibly due to a very slight deviation from axial symmetry). Therefore, the spin Hamiltonian of rhombic symmetry was used to describe the spectrum [37-42]:

$$
H=g \beta \boldsymbol{B} \cdot \boldsymbol{S}+D\left(S_{Z}^{2}-\frac{5}{4}\right)+E\left(S_{X}^{2}-S_{Y}^{2}\right),
$$

where $\beta$ is the Bohr magneton, $\boldsymbol{B}$ is the magnetic inductance vector, $S$ is the operator of electron spin, $\hat{S}_{X}, \hat{S}_{Y}$, $\hat{S}_{Z}$ are spin operators, while $g, D, E$ are the definable parameters.

The results of the data processing and calculation of the parameters of the spin Hamiltonian are

$$
\begin{aligned}
& g=(1.9743 \pm 0.0004), \quad D=(-0.465 \pm 0.001) \mathrm{cm}^{-1}, \\
& E=(-0.013 \pm 0.001) \mathrm{cm}^{-1} .
\end{aligned}
$$

When processing the experimental results, the Easy-spin program was used [43]. As a result, one can see that $g$-factor is almost isotropic and the initial splitting of the spectra is controlled by parameter $D$. In other words, the spectrum is similar to an axial one. Small value of the parameter $E$ indicates that the rhombic distortions around $\mathrm{Cr}^{3+}$ ions are negligible. With using the angular dependence in the vicinity of axis $C_{3}$, the angle of deviation of the $Z$ axis from $C_{3}$ was calculated $\left(1.7^{\circ}\right)$.

To find the sign of parameter $D$, the spectrum was registered at the temperatures of $30 \mathrm{~K}$ and $4 \mathrm{~K}$. Evolution of the relative intensity of the transitions allowed a conclusion that $D<0$ (Fig. 4). Therefore, doublet $\pm 3 / 2$ is of the lowest energy and doublet $\pm 1 / 2$ is located higher by $0.930 \mathrm{~cm}^{-1}$. Splitting of two doublets in zero magnetic field in isomorphic crystals of aluminum borates is almost of the same value, being equal to $1.058 \mathrm{~cm}^{-1}, 0.974 \mathrm{~cm}^{-1}$, and $1.046 \mathrm{~cm}^{-1}$ in $\mathrm{TmAl}_{3}\left(\mathrm{BO}_{3}\right)_{4}, \mathrm{EuAl}_{3}\left(\mathrm{BO}_{3}\right)_{4}$, and $\mathrm{YAl}_{3}\left(\mathrm{BO}_{3}\right)_{4}$, respectively.

The absorption lines associated with the transitions between the levels characterized by unlike quantum numbers differ in width. At the frequency of $9.875 \mathrm{GHz}$, the width of transition $( \pm 1 / 2)$ is 83 Gs, the width of the interdoublet transition is 270 Gs. The width of

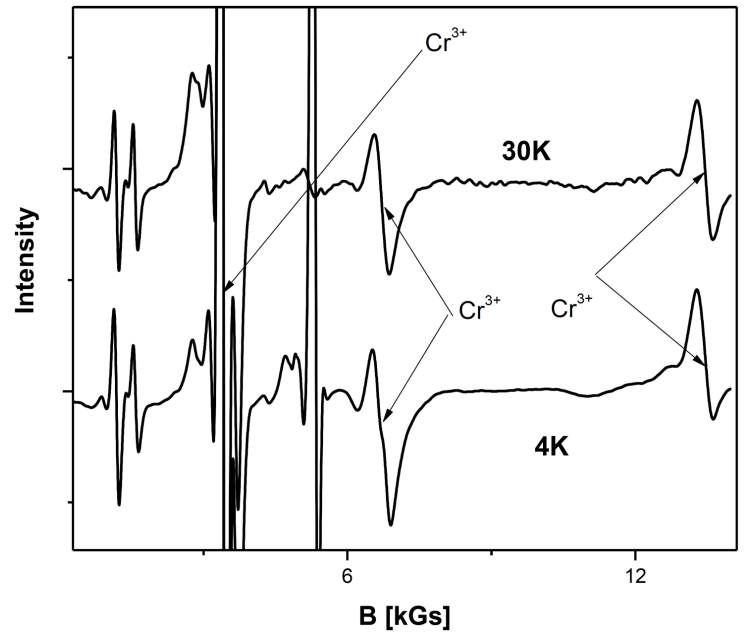

Fig. 4. The EPR spectrum of the $\mathrm{Cr}^{3+}$ ion in a $\mathrm{YGa}_{3}\left(\mathrm{BO}_{3}\right)_{4}$ crystal at a frequency of $f=9.384 \mathrm{GHz}$, $T=30 \mathrm{~K}$, and $4 \mathrm{~K}$. At $T=4 \mathrm{~K}$ less than the intensity of the low-field transition, confirmed that $D<0$.

an absorption line is determined by a number of factors: spin-spin interaction between the chromium ions, electron-nuclear interaction of the $\mathrm{Cr}^{3+}$ ions with the nuclear moments of the neighbors, insignificant changes in the crystal field parameters at various sites, where paramagnetic impurity ions are located, which are taken into account in the Easy-spin program by introducing the DStrain parameter of the crystal field. Spinspin interaction between the chromium ions is insignificant because of small concentration of the doping ions. Electron-nuclear interaction of the $\mathrm{Cr}^{3+}$ ions with the nuclear moments of the neighbors is one of the reasons of central transition broadening $(+1 / 2 \leftrightarrow-1 / 2)$. The environment of a $\mathrm{Cr}^{3+}$ ion includes boron nuclei (the nuclear spin is $3 / 2$, the magnetic moment is equal to $2.688 \mathrm{nu}-$ clear Bohr magnetons, the relative abundance is $80.39 \%$ ) and gallium nuclei $\left(\mathrm{Ga}^{69}\right.$ - the nuclear spin is $3 / 2$, the magnetic moment is 2.01 , the relative abundance is $60.4 \% ; \mathrm{Ga}^{71}$ - the nuclear spin is $3 / 2$, the magnetic moment is 2.56 , the relative abundance is $30.9 \%$ ). It is known that in the crystals of $\mathrm{Al}_{2} \mathrm{O}_{3}$ doped by chromium [34], the width of transition $(+1 / 2 \leftrightarrow-1 / 2)$ is equal to $12 \mathrm{Gs}$ at the minimal dilution. It is obvious that the listed interactions cannot result in the registered broadening. The main reason of the broadening is structural disorder in the crystal lattice, which is reflected in disorder of the crystal field parameters, as well as the zero field splitting ones, thus resulting in dispersion of both the $g$-factor and $D$.

\section{Conclusion}

The crystallographic structure of the $0.1 \%$ Cr-doped $\mathrm{YGa}_{3}\left(\mathrm{BO}_{3}\right)_{4}$ crystallized in the huntite structure were refined. The performed EPR tests of the spectrum of 
the doping ion of $\mathrm{Cr}^{3+}$ in the crystals of $\mathrm{YGa}_{3}\left(\mathrm{BO}_{3}\right)_{4}$ show that an ion of trivalent chromium is positioned at the site of $\mathrm{Al}^{3+}$ in octahedron with rhombic distortions. The spectra of three magnetically nonequivalent ions rotated through $120^{\circ}$ are registered. The parameters of the spin Hamiltonian and the sign of the parameter $D$ are defined.

\section{Acknowledgments}

We acknowledge the Operational Program Research, Development and Education financed by the European Structural and Investment Funds and the Czech Ministry of Education, Youth and Sports (Project No. SAFMATCZ.02.1.01/0.0/0.0/16013/0001406). The research infrastructure SAFMAT was supported by projects LM2015088 and LO1409 by MEYS.

\section{References}

[1] J. Liu, Y. Wan, X. Tian, Z. Zhou, W. Han, J. Li, H. Zhang, J. Wang, Appl. Phys. B 111, 233 (2013).

[2] W. Liang, X.H. Zhang, J. Xia, G.Y. Jin, L.J. Xu, G.C. Sun, Z.M. Zhao, Laser Phys. 21, 861 (2011).

[3] P.A. Burns, J.M. Dawes, P. Dekker, J.A. Piper, J. Li, J.Y. Wang, Opt. Commun. 207, 315 (2002).

[4] A. Brenier, C.Y. Tu, Z.J. Zhu, J.F. Li, Y. Wang, Z.Y. You, B.C. Wu, Appl. Phys. Lett. 84, 16 (2004).

[5] A.D. Balaev, L.N. Bezmaternykh, I.A. Gudim, V.L. Temerov, S.G. Ovchinnikov, S.A. Kharlamova, J. Magn. Magn. Mater. 258, 532 (2003).

[6] M.N. Popova, T.N. Stanislavchuk, B.Z. Malkin, L.N. Bezmaternykh, J. Phys. Condens. Matter 24, (2012).

[7] E.A. Popova, N. Tristan, A.N. Vasiliev, V.L. Temerov, L.N. Bezmaternykh, N. Leps, B. Buechner, R. Klingeler, Eur. Phys. J. B 62, 123 (2008).

[8] M.I. Kobets, K.G. Dergachev, E.N. Khatsko, S.L. Gnatchenko, L.N. Bezmaternykh, I.A. Gudim, Low Temp. Phys. 40, 629 (2014).

[9] K.C. Liang, R.P. Chaudhury, B. Lorenz, Y.Y. Sun, L.N. Bezmaternykh, V.L. Temerov, C.W. Chu, Phys. Rev. B 83, (2011).

[10] A.A. Prokhorov, A.D. Prokhorov, L.F. Chernush, V.P. Dyakonov, H. Szymczak, A. Dejneka, Phys. Scr. 90, (2015).

[11] A.M. Vorotynov, G.A. Petrakovskii, Y.G. Shiyan, L.N. Bezmaternykh, V.E. Temerov, A.F. Bovina, P. Aleshkevych, Phys. Solid State 49, 463 (2007).

[12] C. Rudowicz, P. Gnutek, M. Acikgoz, Opt. Mater. 46, 254 (2015).

[13] M. Acikgoz, P. Gnutek, C. Rudowicz, Opt. Mater. 36, 1342 (2014).

[14] A.A. Prokhorov, L.P. Chernush, V. Babin, M. Buryi, D. Savchenko, J. Lancok, M. Nikl, A.D. Prokhorov, Opt. Mater. 66, 428 (2017).
[15] A.A. Prokhorov, L.F. Chernush, V.P. Dyakonov, H. Szymczak, A.D. Prokhorov, J. Magn. Magn. Mater. 420, 285 (2016).

[16] J.P.R. Wells, M. Yamaga, T.P.J. Han, M. Honda, J. Phys. Condens. Matter 15, 539 (2013).

[17] A.D. Prokhorov, E.E. Zubov, A.A. Prokhorov, L.F. Chernush, R. Minyakaev, V.P. Dyakonov, H. Szymczak, Phys. Status Solidi B 250, 1331 (2013).

[18] A.A. Prokhorov, J. Mater. Sci. 51, 4762 (2016).

[19] M. Acikgoz, C. Rudowicz, P. Gnutek, Opt. Mater. 73, 124 (2017).

[20] V.A. Atsarkin, V. Kravchen, I.G. Matveeva, Sov. Phys. Solid State USSR 9, 2646 (1968).

[21] G. Wang, H.G. Gallagher, T.P.J. Han, B. Henderson, M. Yamaga, T. Yosida, J. Phys. Condens Matter 9, 1649 (1997).

[22] A. Watterich, P. Aleshkevych, M.T. Borowiec, T. Zayarnyuk, H. Szymczak, E. Beregi, L. Kovacs, J. Phys. Condens. Matter 15, 3323 (2003).

[23] A.D. Prokhorov, A.A. Prokhorov, L.F. Chernysh, P. Aleshkevich, V. Dyakonov, H. Szymczak, J. Magn. Magn. Mater. 326, 162 (2013).

[24] A.A. Prokhorov, L.F. Chernush, R. Minyakaev, A. Mazur, T. Zajarniuk, A. Szewczyk, V. Dyakonov, J. Lancok, A.D. Prokhorov, J. Alloys Comp. 765, 710 (2018).

[25] A.D. Prokhorov, I.N. Krygin, A.A. Prokhorov, L.F. Chernush, P. Aleshkevich, V. Dyakonov, H. Szymczak, Phys. Status Solidi A 206, 2617 (2009).

[26] A.D. Prokhorov, A.A. Prokhorov, L.F. Chernysh, V.P. Dyakonov, H. Szymczak, J. Magn. Magn. Mater. 323, 1546 (2011).

[27] A.D. Prokhorov, A.A. Prokhorov, L.F. Chernush, R. Minyakaev, V.P. Dyakonov, H. Szymczak, Phys. Status Solidi B 251, 201 (2014).

[28] A.D. Prokhorov, A.A. Prokhorov, E.E. Zubov, L.F. Chernysh, V. Dyakonov, H. Szymczak, Low Temp. Phys. 40, 730 (2014).

[29] E.L. Belokoneva, L.I. Alshinskaya, M.A. Simonov, N.I. Leonyuk, T.I. Timchenko, N.V. Belov, J. Struct. Chem. 19, 332 (1978).

[30] L.I. Alshinskaya, N.I. Leonyuk, T.I. Timchenko, Krist. Tech. 14, 897 (1979).

[31] E. Beregi, A. Watterich, J. Madarasz, M. Toth, K. Polgar, J. Cryst. Growth 237, 874 (2002).

[32] E.Y. Borovikova, K.N. Boldyrev, S.M. Aksenov, E.A. Dobretsova, V.S. Kurazhkovskaya, N.I. Leonyuk, A.E. Savon, D.V. Deyneko, D.A. Ksenofontov, Opt. Mater. 49, 304 (2015).

[33] N.V. Volkov, I.A. Gudim, E.V. Eremin, A.I. Begunov, A.A. Demidov, K.N. Boldyrev, JETP Lett. 99, 67 (2014).

[34] N.I. Leonyuk, L.I. Leonyuk, Prog. Cryst. Growth Charact. Mater. 31, 179 (1995).

[35] E.L. Belokoneva, A.V. Azizov, N.I. Leonyuk, M.A. Simonov, N.V. Belov, J. Struct. Chem. 22, 476 (1981).

[36] G. Meszaros, E. Svab, E. Beregi, A. Watterich, M. Toth, Physica B 276, 310 (2000). 
[37] C. Rudowicz, M. Karbowiak, Coordin. Chem. Rev. 287, 28 (2015).

[38] C. Rudowicz, S.K. Misra, Appl. Spectrosc. Rev. 36, 11 (2001).

[39] J.R. Pilbrow, M.R. Lowrey, Rep. Prog. Phys. 43, 433 (1980).

[40] A.B. Roitsin, Phys. Status Solidi B 104, 11 (1981).
[41] Multifrequency Electron Paramagnetic Resonance, Ed. S.K. Misra, Wiley-VCH, Weinheim 2011; Erratum, S.K. Misra, C. Rudowicz.

[42] C. Rudowicz, Magn. Res. Rev. 13, 1 (1987); ibid., 13, 335 (1988)

[43] S. Stoll, A. Schweiger, J. Magn. Reson. 178, 42 (2006). 